\title{
Impact of Music Distraction on Dental Anxiety in Children Having Intellectual Disability
}

\author{
Geethanjali Gowdham ${ }^{1}$, Amarshree A Shetty ${ }^{2}$, Amitha Hegde $^{3}$, Lekshmi R Suresh ${ }^{4}$
}

\begin{abstract}
Aim and objective: To analyze the impact of Indian instrumental music on children with intellectual disability (mild) exhibiting dental anxiety during dental procedures using electrical skin resistance measured by a biofeedback machine.

Materials and methods: A total of 20 children of 6-14 years having an intellectual disability (mild) were randomly divided into two groups comprising of 10 each. The study was carried out in a cross-over design, with and without music distraction in two appointments spaced out at a gap of 1 month. The children were subjected to a dental examination, oral prophylaxis, and auditory operative stimuli in both appointments. The electrical skin resistance during each procedure was measured using a galvanic skin response (GSR) biofeedback machine and the values were statistically analyzed using paired and unpaired $t$-tests.

Results: A statistically significant increase in electrical resistance was observed during music distraction, which indicated an anxiety reduction when music distraction was employed.

Conclusion: The increased electrical skin resistance due to low anxiety proves the positive impact of music distraction in intellectually disabled children

Clinical significance: Music can be employed as a distraction technique to reduce anxiety in intellectually disabled children.

Keywords: Dental anxiety, Electrodermal activity, Galvanic skin response, Intellectual disability, Music distraction.

International Journal of Clinical Pediatric Dentistry (2021): 10.5005/jp-journals-10005-1902
\end{abstract}

\section{INTRODUCTION}

Fear and anxiety are said to be the most challenging aspects in pediatric dental practice which cause avoidance of dental care that eventually leads to compromised oral health. It has been reported in the literature that dental setting and dental treatment are the fifth most common cause of anxiety leading to uncooperative behavior which can be managed by pharmacological/nonpharmacological methods of behavior management. Children with intellectual disabilities exhibit greater anxiety due to cognitive impairment making behavior management more difficult due to the problems encountered during communication with the child.

Music distraction has been extensively tried in healthy children and is proved effective. The studies in special children employing the above technique are limited; however, Davila and Menendez have suggested the possibility of employing techniques like a distraction in the mild intellectually disabled group. ${ }^{2}$

Various subjective and objective measures have been highlighted in literature for quantifying anxiety based on the child's age and intellectual development among which electrodermal activity (EDA) is said to have a high degree of sensitivity when compared to other physiological measures. ${ }^{3}$ It is based on the principle that, in the presence of emotional stimuli, the sweat secretion is altered as a result of which changes are produced in the electrical activity of the skin. ${ }^{4}$

The present study was an effort to assess the effectiveness of instrumental music as a distraction technique in mild intellectually disabled children by measuring the EDA of the skin.

\section{Materials and Methods}

Twenty children with mild intellectual disability (having an I.Q. score between 50 and 69) aged between 6 years and 14 years of both genders reporting to NITTE Special Child Care Centre (NSPECC),

\begin{abstract}
${ }^{1-4}$ Department of Pediatric and Preventive Dentistry, $A B$ Shetty Memorial Institute of Dental Sciences, NITTE (Deemed to be University), Mangaluru, Karnataka, India

Corresponding Author: Amarshree A Shetty, Department of Pediatric and Preventive Dentistry, $A B$ Shetty Memorial Institute of Dental Sciences, NITTE (Deemed to be University), Mangaluru, Karnataka, India, Phone: +91 9845353251, e-mail: dramarshreeshetty@nitte. edu.in

How to cite this article: Gowdham G, Shetty AA, Hegde A, et al. Impact of Music Distraction on Dental Anxiety in Children Having Intellectual Disability. Int J Clin Pediatr Dent 2021;14(1):170-174.

Source of support: NITTE Special Child Care Centre (NSPECC), NITTE (Deemed to be University)

Conflict of interest: None
\end{abstract}

a unit of Department of Pediatric and Preventive Dentistry, AB Shetty Memorial Institute of Dental sciences, NITTE (Deemed to be University) were selected by convenience sampling for the study. Ethical clearance was obtained from the Institutional Ethical Committee and informed consent was obtained from the parents/ guardian of the special children.

\section{Inclusion Criteria}

- Children with mild intellectual disability as specified by the Diagnostic and Statistical Manual of Mental Disorders-V (DSM-V) classification given by the American Psychiatric Association. ${ }^{5}$

- Hearing acuity within normal limits.

\section{Exclusion Criteria}

- Children under anti-anxiety medication and anti-cholinergic medication. 
- Children with hearing impairment.

- Uncooperative children.

- Children for whom informed consent was not provided by parents/guardians.The children were randomly divided into two groups, groups I and II comprising of 10 children each. The study was carried out in two appointments for both the groups with a crossover design with an interval of 1 month between appointments. The rationale behind the duration was to ensure that acclimatization at the first visit had minimal impact on the children's dental anxiety in the dental operatory during the second visit. Also, the shorter time interval would facilitate minimal attrition of study subjects in between the two appointments.The first appointment was with music distraction for group I and without music distraction for group II. The second appointment was without music distraction for group I and with music distraction for group II. Each appointment involved,

- Examination using dental hand instruments (mouth mirror and CPITN probe).

- Oral prophylaxis using an ultrasonic scaler.

- Operative stimuli (auditory) using handpiece without bur.The appointments were scheduled at mid-morning hours to avoid any bias due to diurnal variations in sweat production. ${ }^{6} \mathrm{~A}$ split-mouth design was used in the study wherein the lower arch was treated in the first visit and the upper arch treated in the second, to maintain a similar working environment for all children during both visits.The music used for distraction was Indian instrumental music by Debanjan Bhattacharjee (Relaxing Sarod-Raasleela based on raga Tilak Kamod) on a mobile phone (which was handheld by the operator) and a headphone was provided to the children to listen to the music.The EDA, which is one of the physiological measures of anxiety, was measured using a galvanic skin response (GSR) biofeedback machine (Mohan's Electronics and Illuminators) (Fig. 1). Galvanic skin response biofeedback machines can record either the electrical resistance/conductivity of the skin. The equipment used in this study recorded the electrical resistance of the skin. So, an increase in anxiety would decrease the electrical resistance whereas if there is a reduction/absence of anxiety the resistance would be increased. The machine comprised of:

- A pair of copper electrodes can be secured to the palmar aspect of the forefinger and middle finger using Velcro straps (Fig. 2A).

- A digital readout which indicated the EDA (Electrical resistance in Kilo-Ohms)

- A plug to be connected to the power source.

- An earth wire to prevent electrical mishaps.

The GSR values were noted after an initial adaptation period of 5 minutes to familiarize the child with the GSR device to avoid any anxiety caused due to the device itself. The values were noted:

- At baseline.

- During dental examination.

- During oral prophylaxis.

- During auditory operative stimuli.

Galvanic skin response biofeedback provides instantaneous real-time values. It is a well-known fact that dental fear, anxiety, and phobia are a continuum with various physiological responses at each time point. So to minimize the risk of measurement error, three values were measured during each of the above-mentioned procedure and the mean value of those were considered as the GSR value for that particular procedure. Three consecutive values

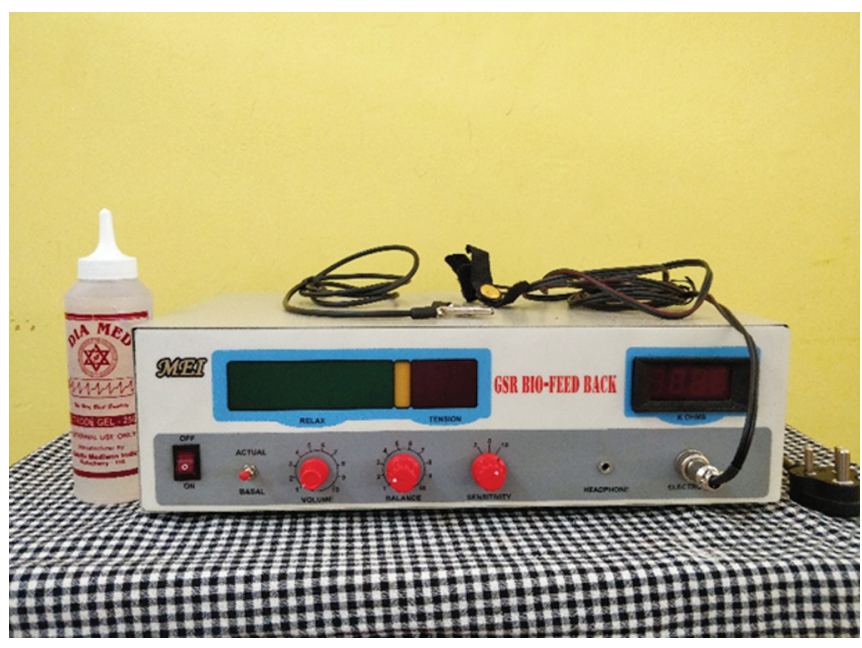

Fig. 1: GSR biofeedback machine

were recorded after the initial adaptation period of 5 minutes and the mean was considered as the baseline value.

During a dental examination, the first value was recorded when the mouth mirror was introduced into the oral cavity, the second after the maxillary arch was examined, and the third was recorded after the mandibular arch was examined (Fig. 2B).

During oral prophylaxis, the first value was noted when the ultrasonic scaler was introduced into the oral cavity, the second when the labial surface was cleaned, and the third value when the lingual surface was cleaned.

For auditory operative stimuli, the first value was noted when the handpiece was operated while it was still in the dental chair, the second was noted when the patient's mouth was closed but the handpiece was held close to the patient's mouth, and third value when it was introduced into the patient's mouth.

The first examiner performed all the procedures during both appointments and the second examiner noted the GSR values (Fig. 3). Verbal cues were used to indicate the recording of values. The mean value of GSR during each procedure in both the appointments were calculated, compared, and statistically analyzed using SPSS software. For quantitative variables, paired $t$-test was used for intragroup comparison and an unpaired $t$-test was used for intergroup comparison. The level of statistical significance was set at a $p$ value of $<0.05$.

\section{Results}

The present study was aimed to evaluate the changes in EDA associated with dental anxiety in children with mild intellectual disability aged between 6 years and 14 years during dental procedures with and without music distraction.

The baseline GSR values were obtained for both groups in both appointments. At baseline, group I was found to have a GSR value of $255.93 \pm 159.509$ in the first visit and $133.83 \pm 93.889$ in the second visit. Group II was found to have a GSR value of $99.63 \pm 57.019$ in the first visit and $268.13 \pm 126.617$ in the second visit.

During the dental examination, the intragroup comparison showed that in group I, the mean GSR values were found to be $318.89 \pm 204.998$ in the first appointment (with music distraction) and $133.93 \pm 77.074$ in the second appointment (without music distraction). In group II, the mean GSR values were found to be $144.93 \pm 54.589$ in the first appointment (without music 



Figs 2A and B: (A) Electrodes with velcro straps secured onto the palmar aspect of distal phalanx of the forefinger and middle finger; (B) Examination carried out with music distraction

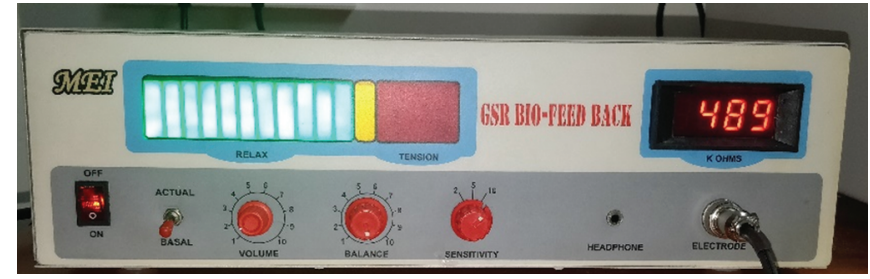

Fig. 3: Recording the electrodermal activity

distraction) and $329.76 \pm 195.024$ in the second appointment (with music distraction). Both the paired $t$-test values were found to be statistically significant. On intergroup comparison, during dental examination in the first appointment in group I (with music distraction) and group II (without music distraction) the GSR values were $318.89 \pm 204.998$ and $144.93 \pm 54.589$, respectively. In the intergroup comparison of the second appointment during a dental examination, the mean GSR values were found to be $133.93 \pm$ 77.074 in group I (without music distraction) and $329.76 \pm 195.024$ in group II (with music distraction). Both the unpaired $t$-test values were statistically significant (Table 1).

The intragroup comparison during oral prophylaxis showed that in group I, the mean GSR values were found to be $369.43 \pm 216.519$ in the first appointment (with music distraction) and $149.73 \pm 68.996$ in the second appointment (without music distraction). Whereas in group II, the mean GSR values were found to be $143.43 \pm 84.244$ in the first appointment (without music distraction) and $417.96 \pm 194.142$ in the second appointment (with music distraction). Both the paired $t$-test values were statistically significant. In the intergroup comparison in the first appointment during oral prophylaxis, the mean GSR values were found to be $369.43 \pm 216.519$ in group I (with music distraction) and $143.43 \pm 84.244$ in group II (without music distraction). In the second appointment during oral prophylaxis, the mean GSR values were found to be $149.73 \pm 68.996$ in group I (without music distraction) and $417.96 \pm 194.142$ in group II (with music distraction). Both the unpaired $t$-test values were statistically significant (Table 2).

The intragroup comparison of GSR values during operative stimuli (auditory) in group I, were found to be $415.99 \pm 243.427$ in the first appointment (with music distraction) and 142.09 \pm 69.700 in the second appointment (without music distraction). In group II, the mean GSR values were found to be $163.23 \pm 131.530$ in the first appointment (without music distraction) and $468.63 \pm 234.707$ in the second appointment (with music distraction). Both the paired $t$-test values were statistically significant. Intergroup comparison revealed that the mean GSR values during operative stimuli (auditory) in the first appointment in group I (with music distraction) and group II (without music distraction) were $415.99 \pm 243.427$ and $163.23 \pm 131.530$, respectively. In the second appointment, the mean GSR values were found to be $142.09 \pm 69.700$ in group I (without music distraction) and $468.63 \pm 234.707$ in group II (with music distraction) (Table 3).

\section{Discussion}

Nonpharmacological methods of behavior management are preferred over pharmacological methods as they are relatively simple, economical, and feasible. However, in children with cognitive impairment, nonpharmacological methods are ineffective making pharmacological methods (in particular general anesthesia) a preferred method of management. The disadvantages however are increased treatment cost, intake of medication, and hospital admission. Since children with intellectual disabilities have been found to have a liking for music, it can be considered as an option to manage dental anxiety in special children. ${ }^{7}$

Literature states that excitatory music increases anxiety whereas calming, non-lyrical, low tone, wind, and string instruments produce a soothing effect during dental treatment for patients. ${ }^{8-10}$ The easy availability and the cultural acceptance of Indian instrumental music make it the best option for our study. It has been reported that the choice of music if given to the children would result in better control over the unpleasant stimulus (dental procedure). ${ }^{11}$ But since our study subjects were intellectually disabled, the decision-making could not be done by them due to which, the same Indian instrumental music was applied to all study subjects of the current study.

The effectiveness of any behavior management is determined by measuring anxiety levels. Anxiety levels measured using physiological parameters are preferred in children with intellectual disabilities over self-reported measures of anxiety. ${ }^{5}$ Several physiological parameters such as blood pressure, heart rate, pulse rate, oxygen saturation, salivary cortisol levels, skin conductance, 
Impact of Music Distraction on Dental Anxiety in Children Having Intellectual Disability

Table 1: Comparison of galvanic skin response levels during dental examination

\begin{tabular}{llllrll}
\hline Group & Appointment & $n$ & Mean & \multicolumn{1}{c}{ SD } & Paired $t$-test & Unpaired $t$-test \\
\hline Group I & 1 & 10 & 318.89 & 204.998 & $t=3.095, p$ value $=0.013$ & $t=3.041, p$ value $=0.007$ \\
& 2 & 10 & 133.93 & 77.074 & & $t=-2.953, p$ value $=0.008$ \\
Group II & 1 & 10 & 144.93 & 54.589 & $t=-3.827, p$ value $=0.004$ & $t$ \\
& 2 & 10 & 329.76 & 195.024 & & \\
\hline
\end{tabular}

Table 2: Comparison of galvanic skin response levels during oral prophylaxis

\begin{tabular}{llllrll}
\hline Group & Appointment & $n$ & Mean & \multicolumn{1}{c}{$S D$} & Paired $t$-test & Unpaired $t$-test \\
\hline Group I & 1 & 10 & 369.43 & 216.519 & $t=3.532, p$ value $=0.006$ & $t=3.076, p$ value $=0.006$ \\
& 2 & 10 & 149.73 & 68.996 & & $t=-4.117, p$ value $=0.001$ \\
Group II & 1 & 10 & 143.43 & 84.244 & $t=-6.194, p$ value $=0.000$ & \\
& 2 & 10 & 417.96 & 194.142 & & \\
\hline
\end{tabular}

$p$ value $\leq 0.05$ is significant, $p$ value $>0.05$ is not significant

Table 3: Comparison of galvanic skin response levels during auditory operative stimuli

\begin{tabular}{llllrll}
\hline Group & Appointment & $n$ & Mean & \multicolumn{1}{l}{$S D$} & Paired $t$-test & Unpaired $t$-test \\
\hline Group I & 1 & 10 & 415.99 & 243.427 & $t=4.305, p$ value $=0.002$ & $t=2.889, p$ value $=0.009$ \\
& 2 & 10 & 142.09 & 69.700 & & $t=-4.217, p$ value $=0.000$ \\
Group II & 1 & 10 & 163.23 & 131.530 & $t=-6.003, p$ value $=0.000$ & $t$ \\
& 2 & 10 & 468.63 & 234.707 & & \\
\hline
\end{tabular}

$p$ value $\leq 0.05$ is significant, $p$ value $>0.05$ is not significant

temperature, electromyography (EMG), electrocardiogram (ECG), and electroencephalography (EEG) have been frequently employed as the measure of dental anxiety in healthy children. ${ }^{12-15}$ The use of EDA, however, as a physiological measure in both healthy and special children is very limited. Studies were done by Davila et al. in intellectually disabled children ${ }^{10}$ and Ferguson et al. in autistic children ${ }^{16}$ are the only studies available in literature utilizing EDA as a measure of anxiety in special children. The above-mentioned observations have inspired us to attempt the use of EDA as a measure of anxiety during dental procedures with music distraction in intellectually disabled children.

The current study was carried out on 20 intellectually disabled children aged between 6 years and 14 years who were subjected to music distraction during dental procedures in a cross-over design in two appointments.

The choice of procedures were dental examination, oral prophylaxis, and operative stimuli (auditory) because the examination is the preliminary step in any dental appointment. This is usually followed by oral prophylaxis. Various authors have stated that children feel anxious when they listen to the sound of a dental handpiece due to which the auditory operative stimulus was included. ${ }^{10}$

During both the appointments when the dental examination was carried out it was found that the mean GSR values in the presence of music distraction were higher when compared to those obtained without music distraction. The anxiety levels were decreased when music distraction was employed which led to decreased sympathetic sweating, thereby leading to increased electrical resistance. Intragroup and intergroup comparisons revealed that the differences observed were statistically significant indicating that music distraction did reduce the dental anxiety associated with a dental examination. This result is in agreement with the study done by Davila et al. in which music was successful in reducing anxiety in intellectually disabled children. ${ }^{10}$

The statistically significant reduction in the anxiety levels noted during oral prophylaxis when music distraction was employed was contradictory to the observations of the study done by Chaturvedi et al. where audio-visual distraction did not affect dental anxiety. ${ }^{11}$

On application of music distraction during auditory operative stimuli, the lower levels of anxiety noted are in accordance with the findings of the study done by Chaturvedi et al. and Marwah et al. who highlighted the success of music distraction when applied during dental procedures in healthy children. ${ }^{11,17}$ The findings of Aitken et al. were contradictory to the results of our current study as they stated that the presence or absence of music distraction displayed no difference in anxiety levels during a restorative procedure. ${ }^{18}$ The significant reduction of anxiety in our study when music distraction was used proves that music masks the auditory stimuli caused by dental handpieces which is the most common anxiety-provoking stimuli.

The above-discussed observations of our study prove that Indian instrumental music was effective in reducing dental anxiety in intellectually disabled children during dental procedures. The use of EDA as a physiological measure was also a success which paves way for future utilization of the same in studies involving special children.

\section{Limitations of the Study}

- Further studies employing invasive dental procedures are also required to validate the effectiveness of music distraction in reducing anxiety during those procedures.

- Further studies are warranted to understand the duration for which the relaxation provided by music persists. 


\section{Future Recommendations}

- The music employed in the present study was Indian instrumental music which is indigenous to our study population. There is scope for future research in other forms of instrumental music for intellectually disabled children.

- A comparison between the effects of music distraction between healthy children and children with intellectual disabilities would help us understand the biological basis of music distraction.

- Effects of individualized interventions like employing favorite music of the individual/specially composed music should be analyzed further. The impact of age, gender, and ethnicity on music distraction also need to be explored.

- The difference in EDA between different age groups of healthy and special children needs to be evaluated.

\section{Conclusion}

Music proves to be a useful nonpharmacological intervention for anxiety management that is noninvasive with relatively no side effects. The significant advantage is that it can also be coupled with other forms of behavior management techniques without any risk of adverse effects. The following conclusions were drawn based on the results of the present study;

- Music distraction using Indian instrumental music was effective in reducing the anxiety levels in children with mild intellectual disability during all three procedures.

- The reduction in anxiety levels was comparable during all three procedures.

- The EDA, i.e., electrical skin resistance that was assessed in this study was found to be higher (indicating lower anxiety levels) when music distraction was used and the difference was found to be statistically significant.

\section{References}

1. Appukuttan DP. Strategies to manage patients with dental anxiety and dental phobia: literature review. Clin Cosmet Ivestig Dent 2016:8:35-50. DOI: 10.2147/CCIDE.S63626.

2. Davila JM, Menendez J. Relaxing effects of music in dentistry for mentally handicapped patients. Spec Care Dent 1986(1):18-20. DOI: 10.1111/j.1754-4505.1986.tb00943.x.

3. Jimeno FG, Bielsa SY, Fernández CC, et al. Objective and subjective measures for assessing anxiety in pediatric dental patients. Eur $J$ Pediatr Dent 2011;12(4):239-244.
4. Kolodziej M, Tarnowski P, Majkowski A, et al. Electrodermal activity for emotional arousal. Bull Pol Ac Tech 2019;67(4):813-826.

5. American Psychiatric Association. Diagnostic and statistical manual of mental disorders. 5th ed., Washington, DC: American Psychiatric Association; 2013.

6. Najafpour E, Aminabadi NA, Nuroloyuni S, et al. Can galvanic skin conductance be used as an objective indicator of children's anxiety in the dental setting? J Clin Exp Dent 2017;9(3):377-383.

7. Vance WC, Toombs S. A procedure for determining the music preferences of mental retardes. J Music Ther 1966;3(2):57-64. DOI: 10.1093/jmt/3.2.57.

8. Kaempf G, Margaret E, Amodei RN. The effect of music on anxiety. AORN J 1989;50(1):112-118. DOI: 10.1016/s0001-2092(07)67642-x.

9. Nilsson $U$. The anxiety and pain reducing effects of music interventions: a systematic review. AORN J 2008;87(4):780-807. DOI: 10.1016/j.aorn.2007.09.013.

10. Zimny GH, Weidenfeller EW. Effects of music upon GSR of children. Child Dev 1962;33(4):891-896. DOI: 10.2307/1126899.

11. Chaturvedi $S$, Walimbe $H$, Karekar $P$, et al. Comparitive evaluation of anxiety level during the conventional dental procedures with and without the audiovisual distraction eyeglasses in pediatric dental patients. J Int Oral Health 2016;8(11):1016-1022.

12. Rayen R, Muthu MS, Chandrasekhar Rao R, et al. Evaluation of physiological and behavioural measures in relation to dental anxiety during sequential dental visits in children. Indian J Dent Res 2006;17(1):27-34. DOI: 10.4103/0970-9290.29895.

13. Dedeepya P, Nuvvula S, Kamatham R, et al. Behvaioural and physiological outcomes of biofeedback therapy on dental anxiety of children undergoing restorations: a randomized controlled trial. Eur Arch Pediatr Dent 2013;15(2):97-103. DOI: 10.1007/s40368-013-0070-3.

14. Shah HA, Swamy KV, Kulkarni S, et al. Evaluation of dental anxiety and hemodynamic changes (Sympthao-adrenal response) during various dental procedures using smartphone applications $v / s$ traditional behavior management techniques in pediatric patients. Int J Appl Res 2017;3(5):429-433.

15. Shetty V, Suresh LR, Hegde AM. Effect of virtual reality distraction on pain and anxiety during dental treatment in 5-8 year old children. $J$ ClinPediatr Dent 2019;43(2):97-102.

16. Ferguson $\mathrm{BJ}$, Hamlin $\mathrm{T}$, Lantz JF, et al. Examining the association between electrodermal activity and problem behavior in severe autism spectrum disorder: A feasibility study. Front Psychiatry 2019;10(654):1-8. DOI: 10.3389/fpsyt.2019.00654.

17. Marwah N, Prabhakar AR, Raju OS. Music distraction: its efficacy in management of anxious pediatric dental patients. J Indian Soc Pedod Prev Dent 2005;23(4):168-170. DOI: 10.4103/0970-4388.19003.

18. Aitken JC, Wilson S, Coury D, et al. The effect of music distraction on pain, anxiety and behavior in pediatric dental patients. Pediatr Dent 2002;24(2):114-118. 